\title{
Protée
}

\section{Mille ans. Interprétation allégorique et littérale du millénium de l'Apocalypse}

\section{Diane Brouillet}

Volume 27, numéro 3, 1999

L’imaginaire de la fin

URI : https://id.erudit.org/iderudit/030568ar

DOI : https://doi.org/10.7202/030568ar

Aller au sommaire du numéro

\section{Éditeur(s)}

Département des arts et lettres - Université du Québec à Chicoutimi

ISSN

0300-3523 (imprimé)

1708-2307 (numérique)

Découvrir la revue

Citer cet article

Brouillet, D. (1999). Mille ans. Interprétation allégorique et littérale du millénium de l'Apocalypse. Protée, 27(3), 19-28. https://doi.org/10.7202/030568ar
Résumé de l'article

En 431 après Jésus-Christ, l'Église condamne le millénarisme et déclare que la croyance au millénium est une hérésie. Par ce décret, l'Église s'élève contre une conception trop terrestre et trop matérielle de l'idéal chrétien et prend par le fait même une position tant politique que religieuse. L'entreprise de marginalisation du millénarisme est le résultat d'un travail d'interprétation complexe auquel ont participé les plus illustres Pères de l'Église. Nous traiterons ici de l'interprétation de saint Augustin qui, dans De la doctrine chrétienne et dans La Cité de Dieu, échafaude une réflexion visant la liquidation du millénarisme et la proclamation du règne spirituel de l'Église. Cette interprétation de saint Augustin prévaut encore aujourd'hui et permet la distinction entre les groupes catholiques traditionnels et les groupes marginaux adeptes des différentes sectes. 


\title{
M ILLE AN S INTERPRÉTATION ALLÉGO RIQ UE ET LITTÉRALE DU MILLÉNIUM DE L'APOCALYPSE
}

\author{
DIANE BRO UILLET
}

\begin{abstract}
Je dis: «Seigneur, quel est ce lieu?» Il me répondit: «C'est la terre de la récompense. N'as-tu pas entendu ce qui est écrit: Bienheureux les doux car ils posséderont la terre. Les âmes des justes sorties de leurs corps sont envoyées pour un temps en ce lieu». Et je dis: "Cette terre sera donc manifestée avant le temps". L'ange me répondit: "Quand le Christ que tu prêches viendra pour régner, la première terre sera détruite par décision divine et la terre promise apparaîtra comme la rosée et les nuages; alors se manifestera le Seigneur JésusChrist, roi éternel: il viendra y habiter avec tous ses saints, il régnera sur eux pendant mille ans et ils se nourriront des choses excellentes que je vais te montrer maintenant». (Apocalypse de Jean, XXI)
\end{abstract}

Si l'on devait retracer les dates qui ont marqué l'histoire du millénarisme chrétien, dont la constituante principale repose d'ailleurs sur un catalogue de dates et de prévisions, on pourrait facilement identifier l'an 431 comme moment charnière. Marquant la condamnation officielle du millénarisme par l'Église, le Concile d'Éphèse, qui eut lieu en cette année, confirma le rejet du chiliasme hors de l'orthodoxie et, désormais, la croyance au millénium était considérée comme une hérésie. Par ce décret, l'Église s'élevait contre la conception terrestre et matérielle de l'idéal chrétien et prenait par le fait même une position politique et religieuse. En insistant sur la dimension spirituelle de la Parousie et du Jugement dernier, l'Église mettait en effet un terme à toute revendication d'un bonheur terrestre et consolidait sa position centralisatrice.

Cette prise de position de l'Église fut légitimée par une stratégie interprétative complexe. En s'appuyant sur la distinction fondamentale entre l'interprétation littérale et l'interprétation allégorique des textes, les Pères de l'Église chrétienne étaient en effet arrivés à liquider le millénarisme chrétien et à réorienter l'idéal du croyant vers l'attente d'un salut céleste et éternel, tout entier déporté vers le futur. Interprétation et pouvoir semblent donc se conjuguer ici, puisqu'il s'agissait pour l'Église de contrer les ambitions visant un bonheur à conquérir et à instituer par l'action positive. En bâillonnant le croyant au nom de la foi, l'objectif de l'Église n'était pas que d'ordre théologique, car elle arrivait du même coup à se prémunir 
contre une certaine partie des critiques visant ses excès et ses abus de pouvoir.

Nous voulons, dans ce qui suit, tenter de cerner l'essence du millénarisme par la négative, c'est-à-dire en examinant le cadre général préexistant à son rejet par l'orthodoxie. À travers une étude des procédures interprétatives de l'herméneutique patristique, il s'agira d'observer la façon dont les textes de l'Apocalypse, et celui de Jean tout particulièrement, ont été lus, interprétés et remis en question à travers un moment spécifique de l'histoire, soit la période qui s'étend des dernières années de l'Antiquité au tout début du Moyen-Âge. L'entreprise de marginalisation du millénarisme s'étend sur plus d'un siècle et elle est le résultat d'un travail de réflexion et d'interprétation auquel ont participé les Pères de l'Église. Nous nous concentrerons ici sur l'interprétation de saint Augustin qui, dans De la doctrine chrétienne et dans La Cité de Dieu, échafaude une réflexion complexe visant à l'extinction du millénarisme et à la proclamation du règne spirituel de l'Église. L'interprétation de saint Augustin, il faut le rappeler, prévaut encore aujourd'hui et permet la distinction entre les groupes catholiques traditionnels et les groupes marginaux adeptes des différentes sectes.

\section{LES PREMIÈRES LECTURES DU TEXTE DE L'APOCALYPSE}

Le débat sur le règne millénaire du Christ a son origine dans ce court extrait de l'Apocalypse de Jean.

Puis je vis des trônes sur lesquels ils s'assirent, et on leur remit le jugement; et aussi les âmes de ceux qui furent décapités pour le témoignage de Jésus et la parole de Dieu, et tous ceux qui refusèrent d'adorer la Bête et son image, de se faire marquer sur le front ou sur la main; ils reprirent vie et régnèrent avec le Christ mille années - c'est la première résurrection. Les autres morts ne purent reprendre vie avant l'achèvement des mille années. Heureux et saint celui qui participe à la première résurrection. La seconde mort n'a point pouvoir sur eux, mais ils seront prêtres de Dieu et du Christ avec qui ils régneront mille années. (Apocalypse de Jean, XX, 4-6)

C'est en effet en référence à ces quelques lignes que les groupes millénaristes ont, de tout temps, légitimé leur croyance en un règne terrestre à venir du Christ et de ses élus. Le lien institué entre le texte de l'Apocalypse et les croyances relatives à ce règne de paix ne fut pas unanimement admis par les premiers commentateurs du texte. Dans l'Antiquité et à l'époque paléochrétienne, il a existé une interprétation du texte qui n'était pas millénariste: la lecture des écrits d'Origène et de Clément d'Alexandrie confirme que l'Apocalypse était lue, commentée et citée par des auteurs qui n'adhéraient pas aux croyances millénaristes et les réfutaient même.

Malgré ces quelques résistances, les croyances millénaristes étaient pourtant très en vogue. Du III au Ve siècle, on retrace ainsi plusieurs auteurs chrétiens adeptes du millénarisme à l'intérieur de l'orthodoxie. Irénée, Hippolyte, Lactance s'appuyaient sur l'Apocalypse de Jean pour affirmer leur croyance et l'on est amené à conclure qu'il existait, comme aujourd'hui d'ailleurs, deux attitudes divergentes au sein du christianisme. Selon une première vision, on était favorable à une interprétation littérale du texte et l'on prônait la croyance à un règne terrestre du Christ d'une durée de mille ans. Le presbytre Gaïus, par exemple, affirmait le caractère chiliaste de l'Apocalypse qui, selon lui, était l'œuvre de Cérinthe, adepte notoire du millénarisme. Dans un sens totalement opposé et fortement soutenu par l'Église officielle à partir du IVe siècle, on a par contre promu une interprétation allégorique du texte et l'on a considéré comme suspecte toute lecture qui s'écartait de ce canon et favorisait l'allégeance au millénarisme.

L'histoire de l'interprétation du texte de l'Apocalypse est probablement l'une des plus riches et des plus complexes que l'on puisse trouver. Il ne saurait être question, pour cette raison, de retracer l'historique de ses interprétations successives. Nous voulons, à tout le moins, tenter de saisir comment la liaison entre millénarisme et Apocalypse en est venue à prendre une telle importance.

Dans la recherche du lien qui unit les croyances millénaristes au texte de l'Apocalypse, un premier fait important à souligner est le caractère morcelé de l'interprétation du texte. Comme plusieurs l'on noté, 
l'interprétation se construit généralement à partir d'éléments isolés, d'abord de leur contexte immédiat, mais également des autres passages du texte où ils apparaissent. En procédant par fragmentation, le processus d'interprétation en vient à se baser sur des symboles et des thèmes qui, par la façon dont ils sont interprétés, semblent vivre d'une vie indépendante et sont analysés pour leur propre compte. Si l'ancienne règle herméneutique voulait qu'on explique le tout en fonction des parties et les parties en fonction du tout, la tradition interprétative de l'Apocalypse s'est démarquée par une procédure qui se concentre sur un élément particulier au détriment de l'ensemble. Il faut souligner que cette procédure n'est pas propre aux seuls «non-spécialistes", mais a été utilisée dans l'ensemble des disciplines concernées par ce texte, touchant autant les ouvrages scientifiques que l'utilisation du texte pour la liturgie ou la théologie.

L'isolation et l'interprétation de certains thèmes spécifiques expliquent la notoriété de certains symboles. Les 4 cavaliers, le $7^{\mathrm{e}}$ sceau, la femme vêtue de soleil, la grande Prostituée, le dragon et la nouvelle Jérusalem constituent les exemples les plus révélateurs. Un thème pourtant se démarque et réapparaît tout au cours de l'histoire et c'est celui des «mille ans». Constituant le cas le plus typique et le plus ancien, il imprime un sens spécifique au texte de l'Apocalypse en ouvrant la voie aux lectures millénaristes.

Il est important de souligner que cette lecture précise du texte n'est pas étrangère à l'Église qui a longtemps promu l'idée d'un millénaire de paix terrestre. Tout au cours de l'histoire, l'orthodoxie catholique s'est en effet approprié différents symboles afin de véhiculer des messages ou des exigences qui la servaient. En «appliquant» ces symboles à une situation particulière, il devenait aisé de promouvoir des visées précises et il en a résulté une cristallisation du sens du texte autour de thèmes qui ont varié au gré des situations. Pour ce qui est de l'interprétation millénariste du chapitre XX de l'Apocalypse, l'Église y trouvait le moyen par excellence pour rallier les chrétiens à sa cause tout en les incitant à résister à l'ennemi.
La date de rédaction du livre de l'Apocalypse correspond en effet à une époque marquée par les persécutions romaines, celle de l'empereur Domitien entre autres. Ce texte, dédié à des chrétiens menacés, se voulait un appel à la résistance, mais aussi et surtout un message de confiance visant à rassurer les chrétiens sur leur foi. Gérard Rochais note à ce titre:

La fonction du prophète est d'édifier, d'exhorter, d'encourager

[...]. Et Jean soutient l'espérance des persécutés, ranime le

courage des tièdes, stimule la conversion des égarés... Lorsque la crise devient à ce point grave qu'elle met en cause l'existence chrétienne elle-même - comme ce fut le cas avec le culte impérial étendu et imposé à tout l'empire sous les Flaviens, notamment sous Domitien - alors il lui faut prêcher la fidélité, la

persévérance et soutenir la constance des fidèles en annonçant la défaite ou la destruction prochaine du persécuteur par quelque force naturelle ou surnaturelle. ${ }^{1}$

En annonçant le retour prochain d'un règne de justice et de paix, le texte de Jean servait directement les intérêts de l'Église qui cherchait à rallier les persécutés et à maintenir leur foi vivante. Écrit pour un auditoire d'opprimés, ce texte permettait d'annoncer le règne prochain de la foi chrétienne et la destruction de Rome, la cité païenne. La présence des thèmes millénaristes n'apparaissait pas d'emblée dangereuse mais une fois les grandes persécutions romaines passées, l'interprétation par trop littérale du texte a commencé à devenir menaçante. Comme l'explique Jean Séguy

[...] le millénarisme s'est répandu et est demeuré vivace tant que la communauté chrétienne a formé un ensemble de groupements persécutés, très minoritaires, rencontrant constamment l'hostilité de la culture globale et de l'état. Par contre, à une plus grande diffusion de cette religion et à sa reconnaissance privilégiée au sein de l'Empire romain a correspondu la marginalisation $d u$ millénarisme à l'intérieur de l'Église. La croyance à un triomphe terrestre du Christ, telle qu'elle apparaît dans l'Apocalypse et dans les écrits millénaristes postérieurs, comportait en effet des éléments non ambigus d'une protestation radicale à l'égard de l'état, de la société et de la culture. L'attitude de l'Église vis-à-vis de ces derniers changea, dès que le christianisme institutionnel acquit quelque respectabilité sociale, et singulièrement lorsqu'il 
devint religion officielle de l'Empire romain. Le triomphe terrestre immédiat de l'Église éliminait la nécessité d'un triomphe à venir du Christ; ce dernier régnait désormais en ce monde par le moyen de l'institution ecclésiastique, assimilée au royaume. De façon significative, le millénarisme devint dès lors une arme idéologique dans la protestation des groupes chrétiens mettant en cause l'État, la culture globale, l'Église elle-même, et les liens historiquement institués entre eux. ${ }^{2}$

Passé la vague des persécutions, les circonstances ont obligé l'Église à réagir contre la montée d'une croyance qui la menaçait indirectement. Le sac de Rome par les barbares a également réactivé les croyances millénaristes, la prise de la Ville éternelle ayant entraîné une vague de peur qui s'est traduite de deux façons différentes. On a assisté à une remise en question de la religion nouvelle, le christianisme, qui n'avait pas su protéger la Ville éternelle contre l'ennemi. Parallèlement, on a noté une recrudescence de l'attente de la fin des temps et de la Parousie, la chute de Rome étant un signe qui ne pouvait tromper. Contre ce fatalisme et le danger de voir les chrétiens se cramponner aux rêves démodés d'un nouveau Paradis terrestre, les Pères de l'Église, et saint Augustin en particulier, ont réagi avec vigueur en décrétant que l'Apocalypse devait être interprétée non à la lettre mais comme une allégorie spirituelle. C'est dans ces circonstances que s'est consumée la séparation entre interprétation littérale et interprétation allégorique et, par conséquent, entre groupes pro et a-millénaristes.

D'une façon paradoxale, il est aussi intéressant de noter que l'Église, en voulant contrer l'interprétation millénariste de l'Apocalypse, n'a fait que renforcer cette lecture. Le travail d'Eusèbe de Césarée a été à cet égard déterminant et a contribué à la cristallisation de l'interprétation sur le thème des «mille ans»:

[...] sa polémique antimillénariste, inspirée par des raisons politiques, a été plus radicale que celle de ses prédécesseurs. Craignait-il de ne plus pouvoir s'opposer à une interprétation millénariste de l'Apocalypse, interprétation que les grandes persécutions contre le christianisme avaient orientée dans un sens fortement antiromain. Quoi qu'il en soit, Eusèbe a contribué à concentrer sur le problème du millénarisme le débat relatif au livre. Depuis lors l'effort des exégètes a été surtout de définir le comment, le quand, la nature de la réalité désignée par le terme $d u$ «règne millénaire». 3

Le travail de saint Augustin est à ce titre révélateur car son ouvrage, La Cité de Dieu, est presque exclusivement consacré au commentaire du chapitre XX de l'Apocalypse, comme si le reste n'existait pas. À l'instar d'Eusèbe de Césarée, saint Augustin condamne les croyances millénaristes et propose une interprétation officielle du texte et du thème du millénium.

\section{ORIGĖNE, PRÉCURSEUR D’AUGUSTIN}

Donc ce huitième jour signifie la vie nouvelle à la fin des temps; le septième jour le repos futur des saints sur le terre. Car le Seigneur régnera en effet sur le terre avec ses saints, comme disent les Écritures, et il aura son Église, où nul ne pénétrera, à l'écart et pure de toute souillure du mal. Car l'Église alors apparaîtra dans une grande clarté, dignité et justice. Là, on n'aura pas de plaisir à tromper, à mentir, à cacher le loup sous la peau de la brebis. En effet le Seigneur viendra comme cela est écrit et il illuminera les mystères des ténèbres et il manifestera les pensées des coeurs et alors chacun louera Dieu... Il n'y aura plus d'injustes, car désormais ils seront séparés.

(saint Augustin, sermon 259)

Bien que l'interprétation officielle adoptée par l'Église soit celle de saint Augustin, il ne fut pas le premier à réagir contre l'interprétation des chiliastes. Tichonius, d'abord, mais surtout Origène au IIIe siècle avait réagi contre l'interprétation littérale du texte.

Considéré comme le fondateur de la science biblique chrétienne au sein de la communauté d'Alexandrie, Origène s'était consacré presque exclusivement à l'exégèse de la Bible. Afin de mener à bien son entreprise, ce commentateur avait rédigé une œuvre colossale basée sur trois types d'interprétation: des scholies, sorte de notes relatives aux passages difficiles, des commentaires développés et enfin des homélies et des sermons. L'exégèse chrétienne, telle que pratiquée à l'époque d'Origène, avait ceci de 
particulier qu'elle s'inspirait largement de la méthodologie philologique et critique du Musée d'Alexandrie. Dans cette optique, le premier devoir de l'exégète était de respecter le texte en se souciant d'abord de l'exactitude littérale. Dans un contexte de mutation des cultures où les sens premiers s'étaient enfouis sous l'épaisseur du temps et les changements de mentalités, le texte ne suffisait toutefois plus et la contribution d'Origène a été de mettre en lumière la difficulté de comprendre le message chrétien devenu de plus en plus impénétrable.

Afin de remédier aux lacunes d'une lecture strictement littérale, Origène avait échafaudé une méthode exégétique qui se distinguait par l'ampleur de son information mais également par l'élan spirituel qui l'habitait et la motivait.

L'Écriture entière, Ancien et Nouveau Testament, a un sens spirituel. [...] Ce sens spirituel ne peut être découvert que par les "spirituels»; il est le fruit de l'ascèse et de la contemplation. C'est pourquoi les Juifs, qui n'ont pas répondu à la grâce du Christ, ne peuvent comprendre que l'Ancien Testament n'est que la figure du Nouveau; c'est pourquoi les gnostiques voient dans l'Ancien Testament l'œuvre du mauvais démiurge, incapables $q u$ 'ils sont d'en saisir le sens spirituel; c'est pourquoi enfin les chrétiens littéralistes se font une fausse idée de Dieu. ${ }^{4}$

La méthode d'Origène avait pour but de saisir la richesse inscrite au cour de la Bible et elle se fondait, pour ce faire, sur une différenciation entre trois sens de l'Écriture, rattachés eux-mêmes aux trois parties de l'homme que sont le corps, l'âme et l'esprit. Dans cette optique, le «sens littéral» est celui auquel restent attachés les simples et les littéralistes, ceux qu'Origène nomme les charnels. Le second sens, moral, recherche l'allégorie qui, derrière la lettre, doit pouvoir édifier la vie morale. Le sens spirituel, enfin, se rapporte aux biens spirituels, c'est-à-dire à la sagesse cachée dans le mystère. Ces sens, qui se répondent les uns les autres par renvois et interférences, sont censés conduire l'âme vers Dieu, dont la présence traverse l'ensemble des Saintes Écritures.

L'importance accordée par Origène au versant spirituel de l'interprétation entraîne l'inévitable condamnation du millénarisme, qui repose sur une interprétation littérale du texte de l'Apocalypse. Origène condamnait globalement le chiliasme en s'en prenant «aux folles inepties et aux conceptions chimériques d'esprits simples qui refusaient le travail intellectuel et préféraient rêver en interprétant les Écritures" 5 .

L'hostilité d'Origène envers les croyances millénaristes a suscité un véritable débat à Alexandrie où Denys, le disciple d'Origène, s'opposait à Corakion, le chef local des millénaristes d'Alexandrie. De cet affrontement résulta une première mise à l'écart officielle du millénarisme, conséquence de la défaite de Corakion. Jean Delumeau rappelle:

Celui-ci, après une discussion de trois jours, avoua sa défaite:

«En présence de tous les pères qui écoutaient, il promit et nous attesta que dans la suite il n'embrasserait plus cette opinion, qu'il n'en discuterait plus, [...], qu'il avait été convaincu». Denys compléta son triomphe en rédigeant une réfutation en règle du millénarisme, le De repromissionibus, où il n'hésitait pas à déclarer apocryphe l'Apocalypse de saint Jean. En fait, le "livre des Révélations» fut maintenu dans le canon, mais non sans difficultés et après le rejet de toutes les lectures trop littérales. Le millénarisme était en voie de marginalisation. 6

Les conséquences du travail d'Origène se mesurent ainsi dans cette première répudiation du millénarisme. Loin de s'arrêter à ce rôle secondaire dans la querelle opposant les adeptes et les détracteurs du millénarisme, Origène a défini dans sa quasitotalité l'espace mental de l'exégèse médiévale et ce pour plus de mille ans. Saint Augustin, qui est son successeur le plus fidèle, a systématisé ses intuitions rectificatives et est arrivé à liquider le millénarisme chrétien primitif.

\section{L'INTERPRÉTATION SPIRITUALISANTE}

DE SAINT Augustin

Passant d'une attitude partisane à une attitude ouvertement hostile au millénarisme, l'évolution de la réflexion de saint Augustin nous permet de saisir la nature des enjeux qui sont au cour du rejet. Dans le sermon 259, rédigé à l'époque où saint Augustin 
adhérait aux croyances millénaristes, l'attente d'un millénaire sabbatique est clairement manifestée:

Après que le vent $d u$ jugement sera passé, la masse des saints apparaîtra brillante de dignité, toute-puissante par ses mérites et se glorifiant de la miséricorde de son libérateur... Depuis la venue du Seigneur, c'est le sixième jour qui s'écoule, nous sommes dans le sixième jour. Et de même que l'homme, d'après la Genèse, a été formé le sixième jour à l'image de Dieu, il est rénové par le Baptême à cette époque qui est comme le sixième jour $d u$ monde pour que nous recevions l'image de notre créateur. Mais quand le sixième jour sera passé, après qu'aura soufflé le vent qui sépare, le repos viendra; et les saints et les justes de Dieu auront leur sabbat. À la suite de ce septième jour, lorsque sur l'aire auront été manifestées ces dignes moissons qui sont la gloire et le mérite des saints, nous nous acheminerons à cette vie et à ce repos dont il est dit qu'aucun oil ne l'a vu, aucune oreille ne l'a entendu... Alors tout reviendra comme au commencement. De même en effet, lorsque sont achevés les sept jours, le huitième est le premier (de la nouvelle semaine), ainsi quand seront terminés et parachevés les sept âges du monde qui passe, nous reviendrons à cette immortalité et à cette béatitude d'où l'homme est déchu. ${ }^{7}$

Par la suite, on sait que saint Augustin abandonnera toutes positions renvoyant au millénarisme, même modéré, et interprétera l'Apocalypse comme signifiant la victoire du Christ dès l'incarnation. Se référant à Tichonius «[Les saints] régneront mille ans, c'est-à-dire dans le siècle présent [...] l'Église est destinée mille ans en ce siècle jusqu'à la fin du monde» ${ }^{8}$, saint Augustin fonde une nouvelle théorie où les «mille ans» sont interprétés comme la période indéterminée dévolue, à partir de l'avènement du Sauveur, au règne de l'Église.

En révisant radicalement sa position initiale, saint Augustin affirme que l'incarnation du Sauveur a fait commencer les «mille ans» de son règne qui sera suivi du Jugement dernier et de l'avènement de la cité céleste qui n'aura pas de fin. En aucun cas, il ne saurait être question d'attendre un millénaire de bonheur dont une certaine interprétation de l'Apocalypse, par trop littérale, tend à vanter les plaisirs. Au sujet des «mille ans» invoqués par Jean, saint Augustin choisit d'en minimiser l'importance et les interprète d'une façon purement symbolique.
Pour les mille ans, on peut les entendre de deux manières. Ou bien parce que ces choses se passent dans les derniers mille ans, c'està-dire au sixième millénaire dont les dernières années s'écoulent présentement. Ces dernières années seront suivies $d u$ sabbat qui n'a point de rois, c'est-à-dire du repos des saints qui n'en finira jamais, de sorte que l'Écriture appelle mille ans la dernière partie de ce temps en prenant la partie pour le tout. Ou bien l'Écriture se sert de ce nombre parfait pour marquer la plénitude du temps. 9

Tout en développant sa réflexion sur l'importance d'une lecture spirituelle du texte, saint Augustin réitère sa croyance en une interprétation faite dans et pour la foi et récuse clairement toute interprétation s'écartant de ce modèle:

De ces deux résurrections Jean l'Évangéliste a parlé dans le livre qu'on appelle Apocalypse de telle manière que la première n'a pas été comprise par certains d'entre nous, et même a été tournée en ridicule par certaines interprétations fallacieuses... Les Millénaristes, à cause de ces mots de ce livre (Apocalypse XX, 1-6) ont supposé une première résurrection corporelle: ils y ont été poussés, entre autres motifs, surtout par ce nombre des mille années, comme s'il convenait qu'un sabbat d'une telle durée prenne place ainsi dans les saints, savoir un tel repose après les six mille ans de labeur depuis le moment où l'homme a été créé et chassé du paradis de félicité pour tomber du fait du péché dans les affres de notre condition mortelle... Six mille ans accomplis sont comme six jours et le septième sera comme un sabbat de mille années suprêmes: pour cette raison les saints ressusciteront pour le célébrer. Cette opinion pourrait être admise si l'on croyait que durant ce septième millénaire les saints jouiront de délices spirituelles $d u$ fait de la présence $d u$ Seigneur, et j'ai été moi-même autrefois de ce sentiment.

Mais comme ceux qui l'adoptent disent que les saints seront occupés de festins charnels et sans limites, en sorte que non seulement ils ne conservent aucune mesure, mais encore dépassent la mesure de ceux mêmes qui n'ont pas le foi, il n'y a, pour être de leur avis, que des âmes charnelles: aussi ceux qui sont spirituels leur ont-ils donné le nom de Chiliastes, du mot grec qui peut se traduire littéralement par millénaristes... 10

Pour comprendre le point de vue de saint Augustin et l'importance qu'il accorde à l'interprétation 
allégorique du texte, il faut observer le cadre qui préexiste à sa réflexion. L'exégèse patristique, telle que saint Augustin la pratique, se développe sur les fondements de la culture alexandrine mais s'en dégage cependant sur certains points, car il est clair que pour les savants de l'époque, prêtres et religieux, recherche de la foi et intellect sont indissociables. Le cercle herméneutique est basé sur l'ultime autorité de l'Église: toute vérité de foi ou de morale est déchiffrée par la reconnaissance de l'Église considérée comme interprète autorisé, et l'on ne peut comprendre le contenu des Écritures que si déjà l'on croit. Le sens se détache ainsi sur le fond d'une précompréhension théologique qui appelle ensuite un travail d'interprétation.

Le mystère des Écritures ne peut donc être atteint par une exégèse stricte des mots. La mise en évidence de leur contenu présuppose au contraire une multiplicité de lectures qui ne saurait épuiser, cependant, le contenu potentiellement infini des textes saints. Dans l'optique de l'exégèse patristique, on note une dévaluation certaine du contenu physique des réalités d'ordre naturel ou historique et la référence essentielle va au transcendant. Toute réalité est en somme affectée d'un coefficient religieux et la prévalence va à la signification qui exprime cette référence et non pas à l'explication qui s'en tient aux causes externes.

Afin d'expliciter cette idée, saint Augustin se réfère à une théorie du langage basée sur la distinction entre signum et res. Dans ce cadre, tout signe est ce qui renvoie à autre chose et il ne peut y avoir qu'une seule chose qui soit pure, qui soit une valeur en soi et qui ne renvoie à rien, c'est le Créateur lui-même. Dans les termes de saint Augustin, seul Dieu est res, tout le reste est signum. Les Écritures sont considérées comme des signes donnés par Dieu aux hommes pour révéler les choses nécessaires au salut.

Du point de vue de l'interprétation, cette règle fait en sorte que tout doit être vu comme signe d'une chose cachée; tout discours expose un ensemble de rappels, de figures et de symboles renvoyant à l'unique Vérité qui est présente derrière chaque mot.
L'interprétation transite bien sûr par le sens littéral mais, comme le remarque G. Gusdorf, «elle ne saurait y faire sa demeure, sans se rendre coupable d'un sacrilège séméiologique» 11 .

S'en tenir délibérément à la "pure lettre", à supposer qu'on le fit avec exactitude, voilà ce qui serait faire œeuvre de sens propre; voilà ce qui serait s'enfoncer dans une interprétation subjective. Ce serait «corrompre l'Écriture» en attentant à son intégrité. Ce serait, en refusant de voir qu'elle rend partout témoignage au Christ, la vider de l'esprit dont elle est pleine, pour la rabaisser au rang des livres humains. 12

Parallèlement à la distinction entre signum et res, saint Augustin propose de distinguer signa propria et signa translata, c'est-à-dire les signes propres, qui sont utilisés pour signifier les choses pour lesquelles ils ont été établis, et les signes figurés qui sont les choses elles-mêmes mais qui, indiquées par des paroles appropriées, signifient autre chose. Il s'agit en somme de la distinction entre sens littéral et sens allégorique, et la tâche de l'interprète est d'établir si l'expression à comprendre a un sens propre ou figuré. Lors du travail d'interprétation, la règle d'or est d'éviter de prendre à la lettre ce qui est dit au sens figuré: «ce serait un misérable esclavage que d'échanger les signes pour la réalité signifiée» ${ }^{13}$. De façon correspondante, on ne doit pas prendre au sens figuré ce qui est dit au sens propre, car sous prétexte d'interprétation allégorique, on peut justifier toutes sortes d'attitudes morales et d'opinions hérétiques.

Afin de mettre en évidence le contenu des Écritures, saint Augustin s'est inspiré du modèle développé par Origène et a proposé de distinguer quatre sens car Dieu,

[...] auteur du texte sacré, possède un intellect infini, capable de comprendre toutes choses en un acte unique, alors que l'homme est obligé d'accumuler des significations fragmentaires, partielles, sans parvenir d'ailleurs à la plénitude de l'intuition divine unitaire. ${ }^{14}$

Sa doctrine des quatre sens distingue d'abord le sens littéral, qui enseigne l'histoire et fournit la clef chronologique de l'intelligibilité judéo-chrétienne. Le 
deuxième sens, dit allégorique, livre ce qu'on doit croire et expose la réciprocité d'action entre l'Ancien et le Nouveau Testament. Comme G. Gusdorf l'explique, ces deux premiers sens

[...] proposent le circuit intellectuel de l'interprétation, système de renvois à l'intérieur du domaine scripturaire. Le sens littéral se limite à la dimension chronologique, selon la succession historique des temps, le sens allégorique pour sa part est anachronique; il établit entre les diverses parties des Écritures une contemporanéité; promesse et accomplissement se correspondent au sein d'un ensemble solidaire, à la fois fermé et ouvert, puisque la Bible chrétienne se termine par le livre de l'Apocalypse, débouché sur un terme eschatologique encore à venir. 15

Conjointement à ces deux sens «objectifs", le sens moral va s'imposer au moment où la Bible est reconnue par le fidèle comme Livre de la Vie, qui sera reçu selon les orientations de sa vie personnelle. Les paroles de Jésus proposent explicitement des règles de vie qui se retrouvent disséminées dans la Bible et le sens moral se situe par conséquent au niveau du dialogue de l'âme fidèle avec Dieu. Prenant forme en dehors de toute référence critique ou rationnelle, ce troisième sens est celui qui permet une transfiguration quotidienne de l'existence selon l'esprit de l'Évangile et selon l'avènement possible du chrétien dans la cité de Dieu.

Le sens anagogique, enfin, est évocateur du mouvement de l'âme vers la transcendance, mais, plus important encore, il met le chrétien en contact avec le sens fondamental de son existence, ce vers quoi elle tend.

Le sens anagogique évoque la perpétuelle annonciation de l'éternité, l'illumination eschatologique de la Présence totale, l'imminence $d u$ grand Retour $d u$ Christ et du dernier jugement. De cet évènement total, Dies irae, dies illa, une lumière nouvelle jaillit, qui situe à leur valeur les préoccupations humaines. Le sens anagogique est le rappel de l'exigence totale du salut, frappant de relativité tout ce qui concerne l'ici-bas, l'en deçà, par rapport à l'au-delà de l'eschatologie. [...] Ce monde-ci, dans sa quotidienneté, mais aussi dans ses luttes et ses combats, n'est que la préparation à un autre monde où la divine Vérité, perceptible ici-bas comme à travers un miroir (per speculum in aenigmate), sera manifestée dans la gloire de sa plénitude accomplie. 16

Dans La Cité de Dieu, saint Augustin réfléchit sur cette idée de préparation à un autre monde et insiste sur la vacuité de l'existence terrestre. Développant un parallèle entre le mythe romain de la cité antique et ce que doit être la cité de Dieu, saint Augustin précise:

Deux amours ont bâti deux cités. L'amour de soi jusqu'au

mépris de Dieu, la cité terrestre. L'amour de Dieu jusqu'au mépris de soi, la cité céleste. L'une se glorifie en elle-même;

l'autre dans le Seigneur. ${ }^{17}$

Dans l'optique de la spiritualité augustinienne, les valeurs terrestres et humaines, telles que préconisées par l'État romain, sont d'abord marquées par leur caractère éphémère et, en ce sens, elles ne devraient pas mériter une trop grande attention de la part du chrétien. Cherchant à contrer les dérives matérialistes du millénarisme, saint Augustin refoule ainsi au second plan l'idée d'un bonheur terrestre et soutient que la vie temporelle n'est que le noviciat de l'éternité.

Afin d'illustrer son propos et de mettre en relief les limites de l'idéal de la cité antique et terrestre, saint Augustin insiste d'abord sur le fait qu'en ne poursuivant que des ambitions terrestres les Romains n'ont reçu que des biens passagers. La Prise de Rome en 410 le prouve. En agissant par patriotisme et en se laissant entraîner par le désir de domination et l'amour de la gloire, les Romains ont récolté le sort qu'eux-mêmes avaient réservé à d'autres, et sont ainsi devenus les victimes de leur propre système.

Pour arriver à ses fins, la cité païenne avait dû maintenir la justice, l'ordre et la paix par l'usage de la force. Or, pour saint Augustin, il n'y a que Dieu qui puisse instaurer cet idéal de paix et de justice. Croyant pouvoir dominer et considérant sa loi et sa paix comme des absolus, la cité romaine s'est annexé, par pur orgueil, des pouvoirs et des vertus qui n’appartiennent qu'à Dieu.

Son ordre et sa justice ne sont finalement que des parodies et une sinistre perversion d'un ordre naturel et chrétien. Ainsi, l'État est 
idolâtre qui, s'érigeant en but suprême, accapare toute l'activité des hommes et prend la place de Dieu, "qui seul dispense la grandeur des empires selon le besoin des temps que sa providence gouverne». 18

À l'encontre de l'idéal purement terrestre d'une cité ayant rejeté Dieu, saint Augustin promeut le modèle de la cité de Dieu qui, éternelle, procurera un bonheur infini aux croyants. Sans être l'exact opposé de la cité antique, la cité de Dieu s'en dissocie cependant sur nombre de principes directeurs. Ainsi, bien qu'elle présuppose aussi le développement de valeurs psychologiques, sociales et culturelles, son existence se trouve cependant traversée de part en part par la présence et la loi de Dieu. La raison de vivre du bon chrétien ne peut donc se résumer dans

l'épanouissement de valeurs humaines au service d'un idéal politique. Si haut que puisse être cet idéal, le bien public ne saurait justifier un tel privilège puisque toute l'existence humaine «se joue en fait dans un arrière-plan qui transcende toute histoire humaine, et où seules comptent les fins surnaturelles» ${ }^{19}$.

Si le citoyen doit apporter sa contribution au monde, il doit cependant garder à l'esprit que les ambitions terrestres sont aussi fugitives que les bonheurs qu'elles engendrent. L'engagement du chrétien dans la communauté doit donc se penser dans les limites prescrites par une allégeance exclusive à la foi chrétienne. Bien que saint Augustin admette qu'il y ait coexistence des deux cités dans le cœur de chaque humain et de chaque société, l'amour de soi et l'amour de Dieu étant inextricablement liés, cette coexistence ne sera cependant jugée positive que dans la mesure où le croyant fera un bon usage des biens terrestres en donnant une priorité à l'amour de Dieu.

Tous les hommes usent des mêmes biens, mais, dans l'usage qu'ils en font, la fin propre diffère selon qu'ils se réfèrent à l'une ou l'autre des cités. Ainsi, mêlés provisoirement durant l'étape temporelle, les citoyens des deux cités se servent des mêmes biens, les uns pour leur salut, les autres pour leur perte. Mais il faut comprendre que ce développement de la cité de Dieu ne peut se réaliser que par l'utilisation du même univers, dans les mêmes cadres sociaux, dans les mêmes structures temporelles que ceux de la cité terrestre. Car, pour Augustin, toute vie, quelle que soit sa destinée, donc son appartenance à l'une ou l'autre des cités, est avant tout sociale... 20

Une vie réussie, telle qu'entendue par saint Augustin, est enracinée dans la création de biens individuels et sociaux sous l'égide de la foi chrétienne qui seule peut conduire le croyant vers ce «sabbat qui n'aura point de soir... vers ce royaume qui n'aura pas de fin ». Henri Desroche note à ce titre:

[...] Augustin transforme l'avènement temporel et abrupt du Royaume des Saints en avènement spirituel et progressif d'une

Église coexistante à la durée du monde. De ce fait, il

dématérialise et détemporalise le jour et en même temps il rend sans objet l'espérance d'une première résurrection. Du fait que, par surcroît, un certain augustinisme, l'augustinisme politique, tendrait à identifier ultérieurement règne de l'Église et chrétienté établie, Augustin lui-même est à peu près considéré comme le liquidateur du millénarisme chrétien primitif. 21

Si certains vont louer l'œuvre de saint Augustin, d'autres relèvent les impacts négatifs de sa pensée.

En répudiant sa foi première au règne des mille ans, il a causé à l'Église un mal inéluctable. Il a sanctionné de l'immense autorité de son nom une erreur qui la privait de son idéal terrestre et qui a fini par plonger les nations chrétiennes dans un désespoir auquel le socialisme veut les arracher à sa manière. 22

Il est vrai que saint Augustin, en concentrant son attention sur un bonheur infini, surnaturel et céleste, accorde peu d'importance au bien-être présent, si temporaire soit-il. La félicité attendue reste, de plus, soumise à des impératifs stricts: le chrétien est encouragé à se préparer à la mort et à son jugement par une vie dépouillée et pleine de bonnes actions. Dans ce cheminement, il demeure dépendant de l'Église institutionnelle considérée comme source de salut et maîtresse d'un enseignement moral dont la sévérité est censée garantir le salut dans l'au-delà. Toute initiative personnelle visant un bonheur trop immédiat devient, dans ce cadre, une forme de contestation et le chrétien est en somme esclave d'un présent qu'il doit supporter en vertu du futur. 
À l'encontre de cette vision des choses, la doctrine millénariste se veut une prise en charge du présent et il ne saurait être question de reporter les «mille ans" de bonheur promis dans un futur lointain. Le millénarisme ne s'entend pas en termes de vie éternelle et de salut des âmes dans un au-delà futur. Au contraire de ce que prône l'Église, on veut le bonheur ici et maintenant, et ce bonheur se traduit généralement par l'avènement d'une société sans classe et d'un règne de justice et de repos pour tous. Le millénarisme concerne l'existence terrestre et implique des actions sociales qui visent à affecter le cours de l'histoire par un appel à l'agir humain.

Selon la définition de Norman Cohn ${ }^{23}$, les différents types de millénarisme partagent certains caractères généraux et constants. Le salut escompté doit d'abord être collectif, les fidèles devant en bénéficier comme collectivité. Terrestre, le salut doit se réaliser sur terre et non dans un paradis appartenant à un autre monde. Le salut doit également être total, c'est-à-dire qu'il doit transformer complètement la vie sur terre. Étant accompli par des agents surnaturels, le salut est enfin miraculeux.

Un de ces caractères entre particulièrement en contradiction avec la doctrine augustinienne reprise par l'Église, et c'est l'idée d'un salut terrestre. Si l'on revient au caractère commun du millénarisme et de l'Église officielle, on peut voir que le millénarisme est en liaison avec la foi chrétienne marquée par le phénomène de l'attente, de l'espérance. Pour comprendre ce phénomène, il faut se rappeler que la religion chrétienne, qui possède une vue linéaire du temps, mobilise l'imagination autour d'une attente, d'une espérance d'un temps de la fin qui apportera le bonheur, le salut dans l'au-delà.

L'espérance propre au millénarisme ne se concentre cependant pas sur un salut lointain et c'est en ce sens que l'adhésion d'un groupe à des croyances millénaristes menace directement l'Église. En permettant aux croyants de se rendre compte de leur situation et d'exprimer leurs insatisfactions, la doctrine millénariste leur confère un statut de revendicateurs qui les sécurise d'emblée, puisque leur avenir apparait désormais lié à leur volonté. L'abdication et le constat d'impuissance disparaissent en somme pour faire place à un investissement concret et à une volonté de changements qui se doivent d'être proches et radicaux. En reconsidérant son rôle dans la société, le chrétien ne peut donc plus suivre les préceptes que lui dicte le modèle strict de la cité de Dieu puisque l'existence présente se voit conférer un statut propre. La conception d'un noviciat sur terre est en somme remplacée par une attitude active où toute soumission et acceptation du sort commun sont rejetées.

La promotion du millénium, issue d'une lecture littérale de l'Apocalypse, ne peut donc qu'être en contradiction avec la doctrine chrétienne qui, en restant attachée à une interprétation allégorique du texte, conçoit la finalité de l'existence humaine en termes d'attente et d'espérance passive.

\section{N O TES}

1. Rochais, G., "Le règne des mille ans et la seconde mort: origine et sens", dans Nouvelle Revue Théologique, 103, nov.-déc. 1981, p. 841.

2. Séguy, J., "Millénarisme", dans Dictionnaire de théologie catholique, Paris, Letouzey et Ané, tome 10, col.1760-1764.

3. Boismard, M.-E., L'Apocalypse, Paris, Éd. du Cerf, 1959, p. 21.

4. Origène, "Euvres. Traité des principes", dans Patrologie grecque, tome XVIII, J.P. Migne (s. la dir. de), Paris, 1857, col. 190.

5. Delumeau, J., Mille ans de bonheur, Paris, Éd. Fayard, 1995, p. 29.

6. Ibid.

7. Saint Augustin, "Sermon 259, § 2 ", dans Patrologie latine, tome XXXVIII, J.P. Migne (s. la dir. de), Paris, 1845-1849, col. 1197.

8. Tichonius, "Commentaires sur l'Apocalyspe, homélie XVIIa ", dans Patrologie latine, tome XXXV, J.P. Migne (s. la dir. de), Paris, 1845-1849, col.2448.

9. Saint Augustin, La Cité de Dieu, tome XX, chap. VII, dans Euvres, trad. G. Combès, Paris, Déclée de Brouwer, 1959-1960, col.666-668.

10. Ibid.

11. Gusdorf, G., Les Origines de l'herméneutique, Paris, Éd. Payot, 1988, p. 72 .

12. Ibid., p. 71.

13. Saint Augustin, La Cité de Dieu, tome III, chap.V, col. 112.

14. Gusdorf, G., op. cit., p. 78.

15. Ibid., p. 81.

16. Ibid., p. 82 .

17. Saint Augustin, La Cité de Dieu, tome XIV, chap. XXVIII, col. 406.

18. Meslin, M., "Augustinisme ", Encyclopedia Universalis, 1989, p. 1113.

19. Ibid., p. 1114.

20. Ibid.

21. Desroche, H., Dieux d'homme, Paris, Éd. Mouton, 1969, p. 56.

22. F. de Rougement, Les Deux Cités, tome 1, Paris, Sandoz et

Fishbacher, 1874, p. 391.

23. N. Cohn, Les Fanatiques de l'Apocalypse, Paris, Payot, 1983. 